\title{
Expression of ER stress markers (GRP78/BiP and PERK) in patients with tongue cancer
}

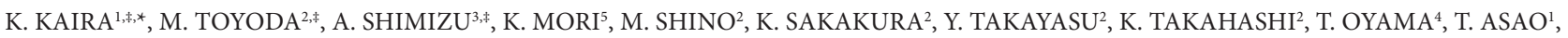 \\ K. CHIKAMATSU ${ }^{2}$
}

${ }^{1}$ Department of Oncology Clinical Development; ${ }^{2}$ Department of Otolaryngology-Head and Neck Surgery; ${ }^{3}$ Department of Dermatology and ${ }^{4}$ Department of Diagnostic Pathology, Gunma University Graduate School of Medicine, Gunma University, Showa-machi, Meabashi, Gunma 371-8511, Japan; ${ }^{5}$ Division of Clinical Trial Coordination Office, Shizuoka Cancer Center, Shizuoka, 411-8777, Japan

*Correspondence: kkaira1970@yahoo.co.jp

*Contributed equally to this work.

\section{Received November 9, 2015 / Accepted February 2, 2016}

\begin{abstract}
The glucose-regulated protein (GRP78/BiP) and PKR-like endoplasmic reticulum kinase (PERK) plays a crucial role in the endoplasmic reticulum (ER) stress response. GRP78/BiP is highly elevated in various human cancers. Our study is to examine the clinicopathological significance of GRP78/BiP and PERK expression in patients with tongue cancer.

A total of 85 tongue cancer patients were analyzed, and tumor specimens were stained by immunohistochemistry for GRP78/BiP, PERK, GLUT1, Ki-67 and microvessel density (MVD) determined by CD34.

GRP78/BiP and PERK were highly expressed in $47 \%$ and $35 \%$ of all patients, respectively. GRP78/BiP disclosed a significant relationship with PERK expression, lymphatic permeation, vascular invasion, glucose metabolism and cell proliferation. The expression of GRP78/BiP was significantly higher in metastatic sites than in primary sites ( $79 \%$ vs. $47 \%, p=0.003$ ). We found that the high expression of GRP78/BiP was proven to be an independent prognostic factor for predicting poor outcome in patients with tongue cancer. In the analysis of PFS, PERK was identified as an independent predictor.

The increased GRP78/BiP expression was clarified as an independent prognostic marker for predicting worse outcome. Our study suggests that the expression of GRP78/BiP as ER stress marker is important in the pathogenesis and development of tongue cancer.
\end{abstract}

Key words: GRP78/BiP, PERK, ER stress, Tongue cancer, prognosis

Tongue cancer includes approximately $30 \%$ of all malignancies of the oral-pharyngeal lesion [1]. This malignant disease is generally treated by surgery, and clinician use disease staging as a most important tool for predicting unfavorable prognosis in patients with tongue cancer [2]. At the time of diagnosis, identifying negative prognostic factors is useful to enable clarification of patients whose tumors have high risk if treatment failure. However, no established biomarker has been elucidated as a predictor after treatment in patients with tongue cancer.

The glucose-regulated protein GRP78, a 78-kDa protein, also referred to as immunoglobulin heavy chain binding protein $(\mathrm{BiP})$, is a major molecular chaperone at the endoplasmic reticulum (ER) that has been characterized on cell membranous and in the cytoplasm (3). GRP78/BiP was involved in the folding and assembly of newly synthesized proteins in the $\mathrm{ER}$ and increased resistance to ER-stress-induced apoptosis (3-5).

The level of GRP78/BiP is highly elevated in many cancer cells and human cancers, and is closely associated with malignancy, metastases and resistance to chemotherapy [4, 5]. There have been only a few studies about the prognostic significance of GRP78/BiP for various patients with breast cancer, lung cancer, gastric cancer, hepatocellular cancer and prostate cancer [5-9].

In oral squamous cell carcinoma (QSCC), it has been reported that the expression of GRP78/BiP was closely associated with tumor size, tumor stage, lymphatic metastasis and distant metastasis, and a positive GRP78/BiP expression was clarified as a significant independent marker for 
predicting poor outcome [6]. Lin et al have described that the overexpression of GRP78/BiP is strongly associated with increasing malignant potential of oral lesions, and is a strong and independent marker for predicting worse outcome of both precancerous and cancerous lesions [7]. On the other hand, it has been shown that the patients with weakly GRP78/BiP expression have a higher incidence of advanced and lymph node metastasis compared to those with strongly GRP78/ $\mathrm{BiP}$ in patients with OSCC [8]. Considering based on these reports, it remains unclear whether the positive expression of GRP78/BiP could predict more unfavorable prognosis than negative expression.

Moreover, PKR-like endoplasmic reticulum kinase (PERK) is considered to be sensors of ER stress [9]. It has been reported that PERK induces apoptosis via CCAAT/enhancer-binding protein homologous protein (CHOP) accumulation under irremediable ER stress, and the inhibition of PERK leads to ER-stress-driven cell death [8]. Vamdexynckel et al documented that the PERK pathway was activated during tumor progression and proapoptotic target $\mathrm{CHOP}$ was upregulated, and a small molecule inhibitor of PERK could be a promising target for cancer therapy [9]. Although GRP78/BiP and PERK have been known as an ER stress marker, we have a controversial opinion whether GRP78/BiP could predict a favorable or unfavorable prognosis after surgery in patients with OSCC, especially tongue cancer [6-8]. In the analysis of patients with oral cancer, the origin from primary sites has not been stratified, thus, these heterogeneity may bias the results of prognostic significance

Here, we conducted the clinicopathological study to evaluate GRP78/BiP and PERK as ER stress related markers for patients with tongue cancer.

\section{Patients and methods}

Patients. Between November 2000 and January 2012, we analyzed 85 consecutive patients with tongue squamous cell carcinoma who underwent surgery at Gunma University Hospital. The authors' approach to the evaluation and resection of these tumors has been described previously [10]. The study was approved by the institutional review board (Ethical Committee approval number: 12-20).

The median age was 69 years, ranging from 33 to 92 years. None had received neo-adjuvant chemotherapy. Formalinfixed, paraffin-embedded primary tumor samples from the 85 patients were examined. Additionally, tissue specimens of cervical lymph-node metastases from 29 patients were available, and one to three tumor-infiltrated lymph nodes were examined in each cases. All surgical specimens were reviewed and classified according to the WHO classification by an experienced pathologist who was unaware of clinical or imaging findings. Pathological tumor-node-metastasis stages were established using the International System for Staging adopted by the American Joint Committee on Cancer and the Union Internationale Centre le Cancer. Postoperative adjuvant chemotherapy with S-1 (Taiho Pharmaceutical Co., Ltd, Tokyo, Japan), oral administration of tegafur (a fluorouracil derivative drug) and docetaxel were administered to 9,12 , and 3 patients, respectively. The day of surgery was considered the starting day for measuring postoperative survival. The follow-up duration ranged from 61 to 3452 days (median, 1033 days).

Immunohistochemical staining. GRP78/BiP and PERK were detected using a rabbit monoclonal antibodies (Cell Signaling Technology, Danvers, MA, 1:100 dilution) and a mouse polyclonal antibodies (Cell Signaling Technology, Danvers, MA, 1:100 dilution), respectively. The detailed protocol for immunostaining has been published elsewhere $[10,11]$. The GRP78/BiP and PERK expression scores were assessed by the extent of staining as follows: $1, \leq 10 \%$ of tumor area stained; $2,11-25 \%$ stained; $3,26-50 \%$ stained; and $4, \geq 51 \%$ stained. The tumors in which stained tumor cells were scored as 3 or 4 were defined as high-expression tumors. Rabbit polyclonal antibody against GLUT1 (AB15309, Abcam, Tokyo, Japan, 1:200 dilution) was used. The expression of GLUT1 was considered positive if distinct membrane staining was present. For GLUT1, a semi-quantitative scoring method was used: $1=<10 \%, 2=10-25 \%, 3=25-50 \%, 4=51-75 \%$ and $5=>75 \%$ of cells positive. The tumors in which stained tumor cells made up more than $25 \%$ of the tumor were graded as high expression.

For CD34 and Ki-67, immunohistochemical staining was performed according to the procedures described in a previous report (10). Mouse monoclonal antibodies against CD34 (Nichirei, Tokyo, Japan, 1:800 dilution) and Ki-67 (Dako, Glostrup, Denmark, 1:40 dilution) were used. The number of CD34-positive vessels was counted in four selected hotspots in a 400X field $\left(0.26 \mathrm{~mm}^{2}\right.$ field area). MVD was defined as the mean microvessel count per $0.26 \mathrm{~mm}^{2}$ field area. The median numbers of CD34-positive vessels were evaluated, and the tumors in which stained tumor cells exceeded the median value were defined as high-expression tumors. For Ki-67, a highly cellular area of the immunostained sections was evaluated. All epithelial cells with nuclear staining of any intensity were defined as high-expression cells. Approximately 1000 nuclei were counted on each slide. Proliferative activity was assessed as the percentage of Ki-67-stained nuclei (Ki-67 labeling index) in the sample. The median value of the Ki-67 labeling index was evaluated, and the tumors exceeding the median value were defined as high-expression tumors. The sections were assessed using light microscopy in a blinded fashion by at least two of the authors.

Statistical analysis. $P$-values of $<0.05$ indicated a statistically significant difference. Fisher's exact test was used to examine the association of two categorical variables. The correlation between different variables was analyzed using the nonparametric Spearman's rank test. The Kaplan-Meier method was used to estimate survival as a function of time, and survival differences were analyzed by the log-rank test. Overall survival (OS) was determined as the time from tumor resection to death from any cause. Progression-free survival 

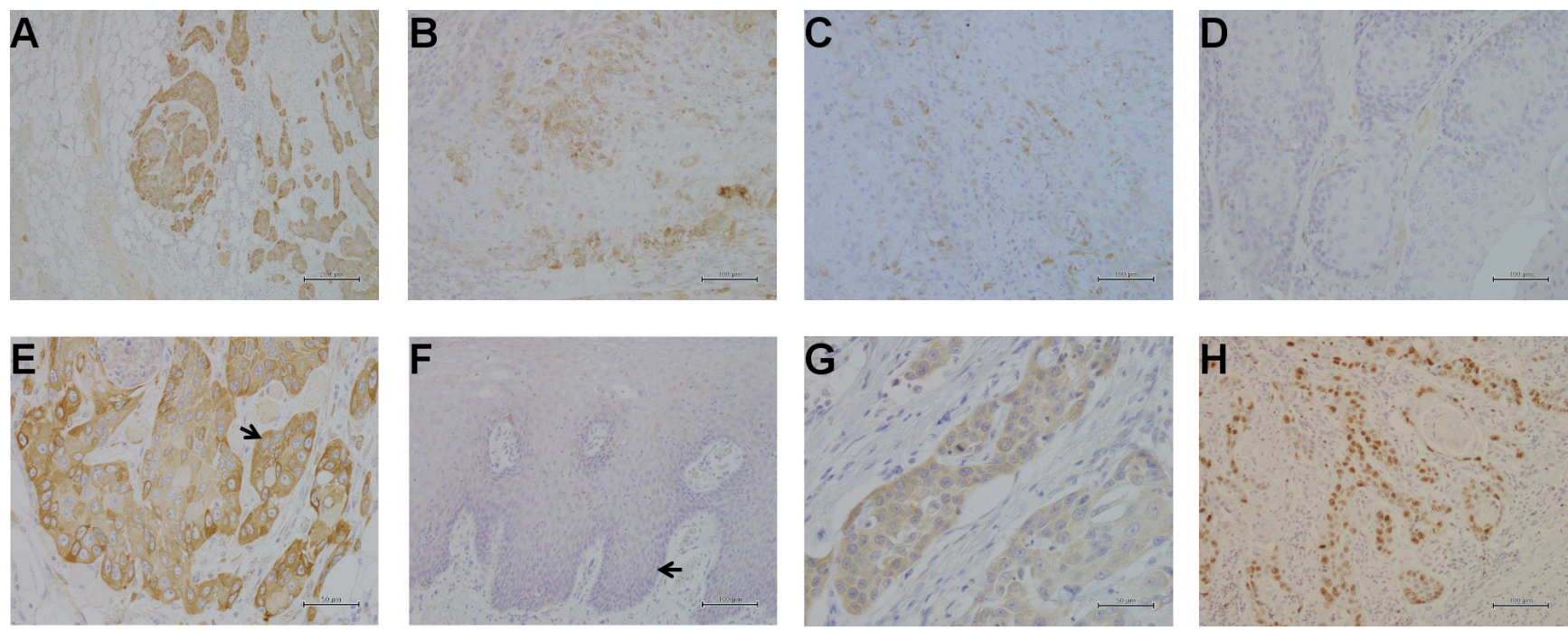

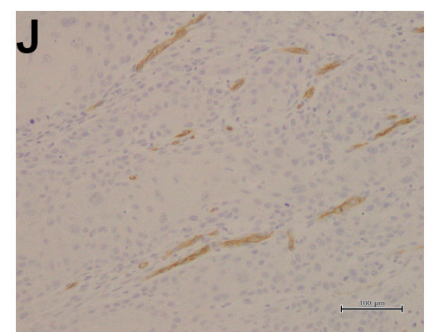

Figure 1. Immunohistochemical staining as representative imaging in patients with tongue cancer. Immunohistostaining of GRP78/BiP [scoring of 4 (A), scoring of 3 (B), scoring of 2 (C) and scoring of 1 (D)] and PERK (G) shows the immunohistostaining pattern of cytoplasm or membrane. Figure 1E shows positive GRP78/BiP staining of malignant tumor cells (black arrow). Figure 1F reveals negative GRP78/BiP staining in normal squamous cells (black arrow). Ki-67 was stained in the nuclei of the tumor cells (H). GLUT1 was stained in the membrane of tumor cells (I). The CD34-positive vessels were stained in tumor tissues $(\mathrm{J})$.

(PFS) was defined as the time between tumor resection and the first disease progression or death. Multivariable analyses were performed using stepwise Cox proportional hazards model to identify independent prognostic factors. Statistical analyses were performed using JMP 8 (SAS, Institute Inc., Cary, NC, USA) for Windows.

\section{Results}

Immunohistochemical staining. The immunohistochemical analyses of different variables were performed on the 85 primary lesions. Figure 1 shows the representative imaging of GRP78/BiP (Figure 1A-1E) and PERK (Figure 1G). GRP78/ $\mathrm{BiP}$ immunostaining was detected in carcinoma cells in tumor tissues and was stained in the cytoplasma and membrane. GRP78/BiP and PERK were highly expressed in 47\% (40/85) and $35 \%(30 / 85)$, respectively, demonstrating no significant difference about the high expression rate of both markers $(p=0.161)$. The median number of CD34-positive vessels was 14 (range, 2-29), and the value of 14 was chosen as a cutoff point (Figure 1J). The median value of the Ki-67 labeling index was $21 \%$ (range, 5-72), which was chosen as the cutoff point (Figure 1H). High CD34, Ki-67 and GLUT1 (Figure 1I) expression was recognized in 52\% (44/85), 51\% (43/85) and $64 \%(54 / 85)$ of patient samples, respectively. Table 1 shows the patient's demographics according to the expression of GRP78/BiP. High expression of GRP78/BIP was significantly associated with lymphatic permeation, vascular invasion, glucose metabolism (GLUT1) and cell proliferation (Ki-67 labeling index).

Next, the immunohistochemical staining of GRP78/ $\mathrm{BiP}$ was performed on the 29 cervical lymph node metastatic sites. In 29 metastatic lymph nodes, GRP78/BiP was highly expressed in $79 \%(23 / 29)$. When the comparison of primary and metastatic carcinomas was done based on paired samples, the expression of GRP78/BiP was significantly higher in metastatic sites than in primary sites ( $79 \%$ vs. $47 \%, p=0.003$ ). Moreover, a statistically significant difference in the scoring of GRP78/BiP was also observed between metastatic and primary sites $(3.1 \pm 0.9$ vs. $2.4 \pm 1.1$, $p=0.001$ ) (Figure 2).

Correlation between GRP78/BiP expression and different variables. Using Spearman's rank correlation, GRP78/ $\mathrm{BiP}$ had a statistically significant correlation with GLUT1 
Table 1. Patient's demographics according to BIP/GPR78 expression

\begin{tabular}{|c|c|c|c|c|c|}
\hline \multirow[b]{2}{*}{ Variables } & & \multirow{2}{*}{$\begin{array}{c}\text { Total } \\
(\mathrm{n}=85)\end{array}$} & \multicolumn{3}{|c|}{ BIP/GRP78 } \\
\hline & & & $\begin{array}{c}\text { High } \\
(\mathrm{n}=40)\end{array}$ & $\begin{array}{c}\text { Low } \\
(\mathrm{n}=45)\end{array}$ & p-value \\
\hline Age & $\leq 65 />65 \mathrm{yr}$ & $36 / 49$ & $14 / 26$ & $22 / 23$ & 0.272 \\
\hline Sex & Male / female & $56 / 29$ & $29 / 11$ & $28 / 18$ & 0.361 \\
\hline Differentiation & $\mathrm{WD}$ or $\mathrm{MD} / \mathrm{PD}$ & $73 / 11$ & $32 / 8$ & $41 / 4$ & 0.213 \\
\hline $\mathrm{T}$ factor & $\mathrm{T} 1-2 / \mathrm{T} 3-4$ & $72 / 13$ & $32 / 8$ & $40 / 5$ & 0.366 \\
\hline $\mathrm{N}$ factor & No / N1-2 & $31 / 54$ & $18 / 22$ & $13 / 32$ & 0.112 \\
\hline Disease stage & I or II / III or IV & $50 / 35$ & $21 / 19$ & $29 / 16$ & 0.279 \\
\hline Ly & yes / No & $31 / 54$ & $22 / 18$ & $9 / 36$ & 0.001 \\
\hline $\mathrm{v}$ & yes / No & $26 / 59$ & $18 / 22$ & $10 / 35$ & 0.037 \\
\hline PERK & High / Low & $30 / 55$ & $17 / 23$ & $13 / 32$ & 0.256 \\
\hline Glut1 & High / Low & $54 / 31$ & $31 / 9$ & $23 / 22$ & 0.014 \\
\hline Ki-67 & High / Low & $43 / 42$ & $29 / 11$ & $14 / 31$ & $<0.001$ \\
\hline $\mathrm{CD} 34$ & High / Low & $44 / 41$ & $23 / 17$ & $21 / 24$ & 0.386 \\
\hline
\end{tabular}

Abbreviation: WD, well differentiated; $\mathrm{MD}$, moderate differentiated; $\mathrm{PD}$, poorly differentiated; ly, lymphatic permeation; $\mathrm{v}$, vascular invasion.

$(r=0.284, p=0.009)$ and $\mathrm{Ki}-67(r=0,554 p<0.001)$, but not CD34 $(r=0.149, p=0.173)$ (Table 2).

Survival analysis. The five-year survival rate and median survival time for all patients were $31 \%$ and 18 months, respectively. We had already reported the detailed information about the survival analysis of various clinicopathological variables and several biomarkers in the previous studies [10]. Table 3 shows the univariate and multivariate analysis in OS and PFS. Figure 3 shows the Kaplan-Meier survival curves for patients with a positive and negative expression of GRP78/BiP (Figure 3A-B) and PERK (Figure 3C-D). Univariable analysis revealed that disease stage, lymphatic permeation, vascular invasion, GRP78/BiP and Ki-67 labeling index were identified to be significant variables for OS, and significant prognostic factors for PFS were differentiation, lymphatic permeation, GRP78/ BiP, PERK and Ki-67 labeling index. Multivariable analysis confirmed that disease stage and GRP78/BiP were independent prognostic factors for predicting negative OS and PFS. In the analysis of PFS, PERK was identified as an independent predictor.

\section{Discussion}

This is a clinicopathological study evaluating the prognostic significance of GRP78/BiP expression in patients with tongue

Table 2. Correlation with BIP/GRP78 expression

\begin{tabular}{lccc}
\hline & Spearman $r$ & $95 \%$ CI & p-value \\
\hline PERK & 0.329 & 0.118 to 0.511 & 0.002 \\
Ki-67 & 0.554 & 0.382 to 0.690 & $<0.001$ \\
CD34 & 0.149 & 0.072 to 0.356 & 0.173 \\
Glut1 & 0.284 & 0.065 to 0.476 & 0.009 \\
\hline
\end{tabular}

Abbreviation: 95\%CI, 95\% confidence interval. cancer. GRP78/BiP was highly expressed in 47\% (40/85), and yielded a significant relationship with lymphatic permeation, vascular invasion, glucose metabolism and cell proliferation. We found that the high expression of GRP78/BiP was proven to be an independent prognostic factor for predicting negative outcome in patients with tongue cancer. Our study suggests that the expression of GRP78/BiP as ER stress marker is associated with the pathogenesis and development of tongue cancer.

Recently, it has been already described that GRP78/BiP is antiapoptotic and plays an important cytoprotective role in oncogenesis. There is a contrary discussion about the prognostic significance of GRP78/BiP expression in various human neoplasms. A high GRP78/BiP expression in patients with hepatocellular, gastric, prostate and renal cell carcinoma achieved a worse prognosis compared to a low expression, whereas, a low GRP78/BiP expression yielded an unfavorable survival in esophageal and lung cancer [5]. In in vitro

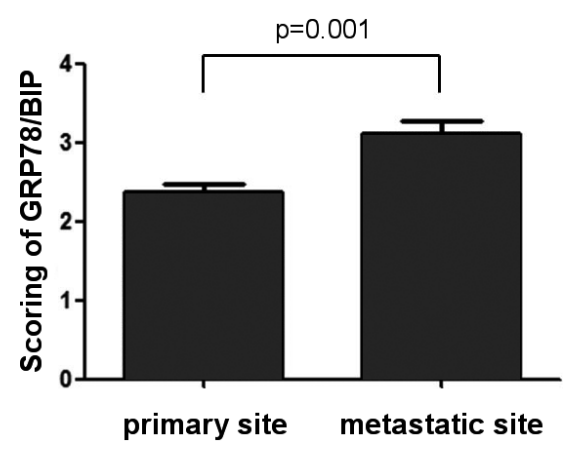

Figure 2. Comparison of scoring of GRP78/BiP expression between metastatic and primary sites. A statistically significant difference in the scoring of GRP78/BiP was observed between metastatic and primary sites $(3.1 \pm 0.9$ vs. $2.4 \pm 1.1, p=0.001$ ). 
A

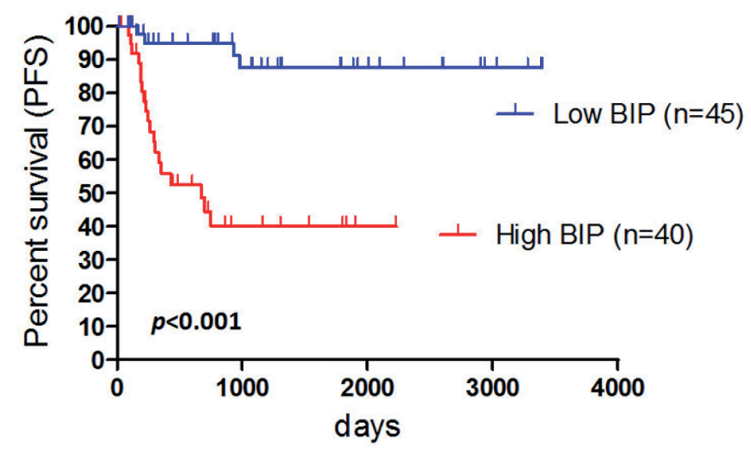

C

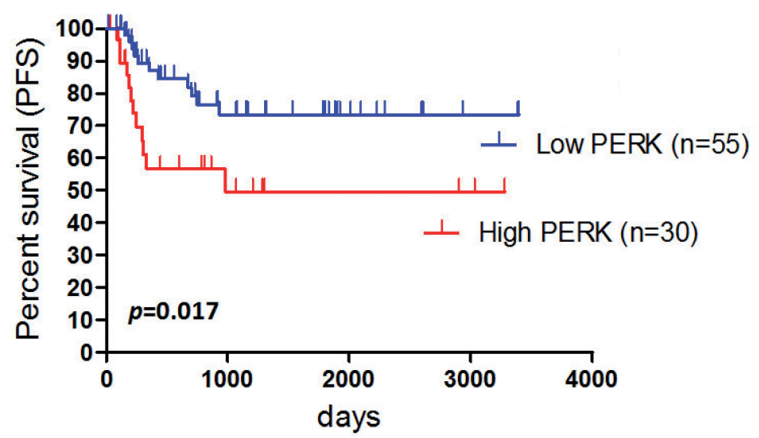

B

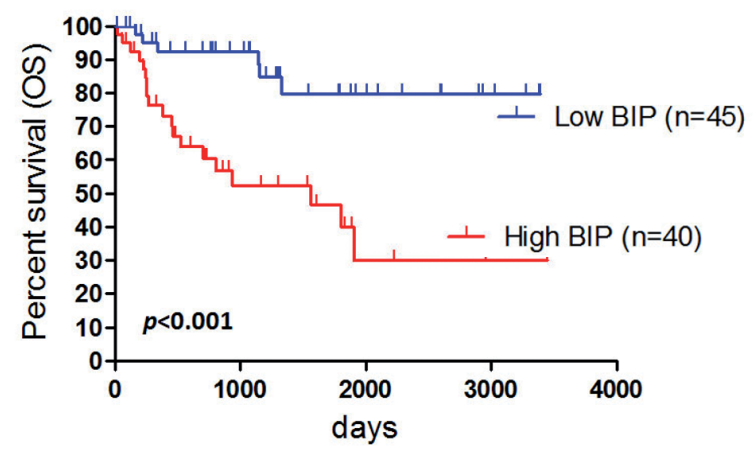

D

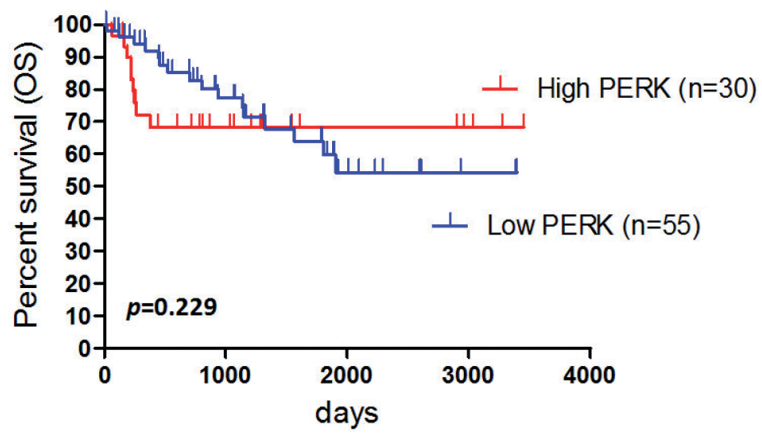

Figure 3. Kaplan-Meier survival curve of overall survival (OS) and progression-free survival (PFS) according to the expression of GRP78/BiP and PERK. A statistically significant difference in OS and PFS was recognized between patients with high and low GRP78/BiP expression [PFS, $p<0.001$ (A); OS, $p<0.001(\mathrm{~B})$ ], and between patients with high and low GRP78/BiP expression [PFS, $p=0.017$ (C); OS, $p=0.229$ (D)], respectively.

studies reported that GRP78/BiP is requiring for the tumor progression and highly metastatic cancer cell lines induced high expression level of GRP78/BiP [12]. In the present study, the protein expression of GRP78/BiP in metastatic lesions was significantly higher than in primary lesions. Our study suggests that the up-regulation of GRP78/BiP expression was observed in the metastatic lesions of individual patients. Lin et al have documented that the overexpression of GRP78/BiP was closely correlated with increasing malignant potential of oral sites, with $14 \%$ in leukoplakia, $27 \%$ in erythroplakia, $50 \%$ in

Table 3. Univariable and multivariable survival analysis in all patients

\begin{tabular}{|c|c|c|c|c|c|c|c|c|c|c|c|}
\hline \multirow{3}{*}{ Variables } & & \multicolumn{5}{|c|}{ Overall survival } & \multicolumn{5}{|c|}{ Progression-free survival } \\
\hline & & \multicolumn{2}{|c|}{ Univariable } & \multicolumn{3}{|c|}{ Multivariable } & \multicolumn{2}{|c|}{ Univariable } & \multicolumn{3}{|c|}{ Multivariable } \\
\hline & & 5 -yrs rate $(\%)$ & $P$-value & HR & $95 \% \mathrm{CI}$ & $P$-value & 5 -yrs rate(\%) & $P$-value & HR & $95 \% \mathrm{CI}$ & $P$-value \\
\hline Age & $\leq 65 />65 \mathrm{yr}$ & $64.8 / 56.3$ & 0.383 & 0.905 & $0.588-1.364$ & 0.635 & $61.8 / 68.3$ & 0.504 & 1.541 & $0.966-2.523$ & 0.069 \\
\hline Sex & Male / female & $58.9 / 71.2$ & 0.847 & 0.913 & $0.587-1.487$ & 0.698 & $62.3 / 72.4$ & 0.585 & 1.245 & $0.707-2.128$ & 0.432 \\
\hline Differentiation & $\mathrm{WD}$ or $\mathrm{MD} / \mathrm{PD}$ & $64.6 / 43.6$ & 0.09 & & & & $40.0 / 37.8$ & 0.037 & & & \\
\hline Stage & I or II / III or IV & $73.4 / 34.2$ & 0.003 & 1.555 & $1.021-2.379$ & 0.04 & $72.1 / 46.9$ & 0.103 & 0.903 & $0.577-1.423$ & 0.655 \\
\hline Primary tumor status & $\mathrm{T} 1-2 / \mathrm{T} 3-4$ & $65.6 / 23.1$ & 0.112 & & & & $66.9 / 36.4$ & 0.785 & & & \\
\hline Lymphatic permeation & Positive/Negative & $39.6 / 74.3$ & 0.007 & & & & $43.7 / 77.7$ & 0.004 & & & \\
\hline Vascular invasion & Positive/Negative & $32.3 / 72.7$ & 0.001 & & & & $50.5 / 70.6$ & 0.14 & & & \\
\hline Resected status & Positive/Negative & $54.5 / 63.0$ & 0.806 & & & & $59.3 / 66.8$ & 0.715 & & & \\
\hline Adjuvant chemotherapy & Yes / No & $40.7 / 71.1$ & 0.217 & & & & $42.3 / 73.3$ & 0.058 & & & \\
\hline GRP78/BIP & High / Low & $39.9 / 79.7$ & $<0.001$ & 1.955 & $1.243-3.298$ & 0.003 & $40.0 / 87.5$ & $<0.001$ & 3.326 & $1.891-6.600$ & $<0.001$ \\
\hline PERK & High / Low & $68.3 / 59.7$ & 0.632 & 0.980 & $0.648-1.527$ & 0.927 & $49.6 / 73.4$ & 0.018 & 1.745 & $1.131-2.722$ & 0.012 \\
\hline $\mathrm{CD} 34$ & High / Low & $48.9 / 75.3$ & 0.182 & & & & $68.7 / 72.1$ & 0.617 & & & \\
\hline Ki-67 & High / Low & $46.8 / 73.3$ & 0.036 & & & & $48.1 / 81.2$ & 0.001 & & & \\
\hline
\end{tabular}

Abbreviation: WD, well differentiated; MD, moderate differentiated; PD, poorly differentiated; HR, hazard ratio; $95 \%$ CI, $95 \%$ confidence interval. 
verrucous lesion, and 74\% in oral cancer [7]. They speculated the essential role of GRP78/BiP expression in the early steps of oral oncogenesis [7]. Our study suggests that GRP78/BiP play a crucial role in the metastatic process of cancer cells. It may contribute to a poor survival as negative predictor in tongue cancer. Further study is warranted to confirm the results of our study by a large-scale study.

Recently, Huang et al have described that decreased GRP78/BiP protein expression was significantly correlated with advanced disease stage and neck lymph node metastasis in patients with OSCC [8]. In their study, the patients with early disease stage disclosed strong GRP78/BiP expression and those with advanced stage showed weak GRP78/BiP expression [8]. Their results suggest that low-grade cancer cells are under ER stress condition and high-grade cancer cells can overcome ER stress-induced apoptosis. On the other hand, Xia et al reported that positive GRP78/BiP expression could act as a prognostic predictor of shorter overall survival [6]. In their study, the increased GRP78/BiP expression was closely associated with advanced stage, lymphatic metastasis, distant metastasis and high grade malignancy. Their study suggests that the patients with positive GRP78/BiP expression disclosed a higher risk of dying from OSCC than those with negative GRP78/BiP expression and play an essential role in the tumor progression and metastasis of OSCC. In these both reports $[6,8]$, but, there have not been clearly described regarding detailed information according to a subtype of OSCC, such as tongue cancer. Moreover, these studies $[6,8]$ have evaluated the prognostic significance of GRP78/BiP expression using approximately 50 patients with OSCC. This is a small sample size, and it may bias the results of these studies. In both these studies $[6,8]$, the same antibody of GRP78/BiP (Santa Cruz Biotechnology, sc-13968) was used, but the evaluation of GRP78/BiP expression was different. Our study was performed by different antibody and evaluation of GRP78/BiP from these studies. Further study is warranted to investigate the clinicopathological significance of GRP78/BiP expression using the same antibody and evaluating methods.

Several descriptions have disclosed that the positive expression of GRP78/BiP is closely associated with metastasis, advanced stage and survival in human cancers $[5,7,8]$, but the expression of GRP78/BiP had not been still examined using the tumor specimens of metastatic sites. Therefore, it remains obscure about the different expression of GRP78/ $\mathrm{BiP}$ between the primary and metastatic sites of OSCC. To our knowledge, our study is a first clinicopathological investigation to evaluate the clinical significance of GRP78/ $\mathrm{BiP}$ expression in tongue cancer using the primary and metastatic sites. Our data supports the relationship between shorter survival and the increased expression of GRP78/BiP in OSCC, especially tongue cancer. Cell surface GRP78/BiP is an upstream regulator of PI3K/AKT signaling. Recent study reported that the knockout of GRP78/BiP exhibited severe reduction of tumor angiogenesis and metastatic growth and GRP78/BiP is a critical mediator of angiogenesis by regulat- ing cell proliferation, migration and survival [13]. The cell surface of GRP78/BiP has been described to be colocalize with proangiogenic growth factor receptors, promoting growth signaling, migration and cell proliferation, and the expression of cell surface GRP78/BiP is induced by vascular endothelial growth factor (VEGF) and needs VEGF-induced proliferation and angiogenic signaling $[14,15]$. That is why GRP78/BiP expression in the primary tumors was closely related to the tumor aggressiveness and progression and the metastatic lesions showed a higher GRP78/BiP expression than the primary lesions.

In a recent review, it has been described that high levels of GRP78/BiP expression correlated with drug resistance, recurrence and poor survival [5]. The down-regulation or inhibition of GRP78/BiP activity may be a potential of molecular target for the treatment of various cancers. This review has described the relationship between GRP78/BiP and drug resistance in various human neoplasms [5]. The induction of GRP78/BiP could be a target therapy against the drug resistant cells of lung cancer, bladder cancer, breast cancer, whereas the inhibition of GRP78/BiP is effective for the resistant cells of gastric cancer, transformed fibroblasts and epidermoid carcinoma. Although there are many discussions regarding the induction or inhibition of GRP78/BiP as a potential of therapeutic target, it is important that we discover any small molecules related to the modulation of ER stress signaling pathway and GRP78/ BiP expression.

Although the expression of PERK plays an important role in the tumor progression as a marker of ER stress, it remains unclear whether a positive or negative PERK expression shows a significant association with the survival and aggressiveness of patients with OSCC $[13,14]$. In the present study, a high PERK expression was clarified as an independent prognostic factor for predicting poor PFS in tongue cancer, and there was a significant correlation between GRP78/BiP and PERK expression. The role of PERK as a prognostic predictor seemed to be markedly weak compared to that of GRP78/BiP, however, our data suggests that both GRP78/BiP and PERK are essential for the development and pathogenesis of tongue cancer. Although we investigated whether a cooperative expression of GRP78/BiP with PERK is a more powerful prognostic marker rather than single marker of GRP78/BiP, we couldn't get a significant finding about the role of these combinations (data not shown). In the present study, we consider that these ER stress markers disclose a significant implication as the assessment of tumor aggressiveness and survival. Further study is warranted to conduct whether GRP78/BiP and PERK could be a novel marker for predicting outcome after chemotherapy or radiotherapy.

There are some limitations of the current study. One limitation is that the sample size in this study was small, which may bias our results. Another limitation is that we focused on the subtype of tongue cancer and didn't investigate the other subtype of OSCC arising from mouth mucosa, maxillary gingiva, mandibular gingiva, and hard palate. Therefore, it 
remains unclear whether our study is completely corresponding to the results of previous studies. Further investigation is warranted to evaluate the prognostic significance of ER stress marker, GRP78/BiP and PERK for the patients with OSCC using a large sample size.

In conclusion, GRP78/BiP was highly expressed in tongue cancer, and our study suggests that GRP78/BiP as ER stress marker is closely related to the tumor aggressiveness and pathogenesis of tongue cancer. By multivariable analysis, the high expression of GRP78/BiP was identified as an independent prognostic factor for predicting negative outcome. However, the prognostic significance of PERK expression seemed to be weak in compared with that of GRP78/BiP expression. ER stress marker, GRP78/BiP could be a promising molecular target for the treatment of tongue cancer.

Acknowledgements: We appreciate Ms. Yuka Matsui for her technical assistance of manuscript submission and Ms. Tomoko Okada for her data collection and technical assistance. We also deeply appreciate Prof. Masahiko Nishiyama of Department of Molecular Pharmacology and Oncology, Gunma University Graduate School of Medicine, for the critical review of this manuscript.

\section{References}

[1] REGEZI JA, SCIUBBA JJ, JORDAN RCK. Oral pathology: clinical, pathologic correlation. 5th ed. St Louis (MO): 2008; Saunders Elsevier.

[2] BELlO IO, SOINI Y, SALO T. Prognostic evaluation oral tongue cancer: Means, markers and perspectives (I). Oral Oncol 2010; 46: 630-5. http://dx.doi.org/10.1016/j. oraloncology.2010.06.006

[3] HENDERSHOT LM. The ER function $\mathrm{BiP}$ is a master regulator of ER function. Mt Sinai J Med 2004; 71: 289-97.

[4] GAZIT G, LU J, LEE AS. De-regulation of GRP stress protein expression in human breast cancer cell lines. Breast Cancer Res Treat 1999; 54: 135-46. http://dx.doi.org/10.1023/ A:1006102411439

[5] LI J, LEE AS.: Stress induction of GRP78/BiP and its role in cancer. Curr Mol Med 2006; 6: 45-54. http://dx.doi. org/10.2174/156652406775574523

[6] XIA F, XU JC, ZHANG P, ZHANG YY, ZHANG QW, et al. Glusose-rekated protein 78 and heparanase expression in oral squamous cell carcinoma: correlation and prognostic significance. World J Surg Oncol 2014; 12: 121. http://dx.doi. org/10.1186/1477-7819-12-121

[7] LIN CY, CHEN WH, LIAO CT, CHEN IH, CHIU CC, et al. Positive association of glucose-related protein 78 during oral cancer progression and the prognostic value in oral precan- cerous lesions. Head Neck 2010; 32: 1028-39. http://dx.doi. org/10.1002/hed.21287

[8] HUANG TT, CHEN JY, TSENG CE, SU YC, HO HC, et al. Decreased GRP78 protein expression is a potential prognostic marker of oral squamous cell carcinoma in Taiwan. J Formos Med Assoc. 2010; 109: 326-37. http://dx.doi.org/10.1016/ S0929-6646(10)60060-5

[9] VANDEWYNCKEL YP, LAUKENS D, BOGAERTS E, PARIDAENS A, VAN DEN BUSSCHE A, et al. Modulation of the unfolded protein response impedes tumor cell adaptation to proteotoxic stress: a PERK for hepatocellular carcinoma therapy. Hepatol Int 2015; 9: 93-104. http://dx.doi. org/10.1007/s12072-014-9582-0

[10] TOYODA M, KAIRA K, OHSHIMA Y, ISHIOKA NS, SHINO M, et al. Prognostic significance of Amino Acid Transporter Expression (LAT1, ASCT2 and xCT) in Surgically Resected Tongue Cancer. Br J Cancer 2014; 110: 2506-13. http://dx.doi.org/10.1038/bjc.2014.178

[11] KAIRA K, TOYODA M, SHIMIZU A, SHINO M, SAKAKURA $\mathrm{K}$, et al. Expression of ER stress markers (GRP78/BiP and PERK) in adenoid cystic carcinoma. Acta oto-Laryngologica 2015; 136: 1-7. http://dx.doi.org/10.3109/00016489.2015.1083120

[12] RAMSAY RG, CIZNADIJA D, MANTAMADIOTIS T, ANDERSON R, PEARSON R. Expression of stress response protein glucose regulated protein-78 mediated by c-Myb. Int J Biochem Cell Biol 2005; 37: 1254-68. http://dx.doi. org/10.1016/j.biocel.2004.12.011

[13] DONG D, STAPLETON C, LUO B, XIONG S, YE W, et al. A critical role for GRP78/BiP in the tumor microenvironment for neovascularization during tumor growth and metastasis. Cancer Res 2011; 71: 2848-57. http://dx.doi. org/10.1158/0008-5472.CAN-10-3151

[14] SHANI G, FISCHER WH, JUSTICE NJ, KELBER JA, VALE W, et al. GRP78 and Cripto form a complex at the cell surface and collaborate to inhibit transforming growth factor beta signaling and enhance cell growth. Mol Cell Biol 2008; 28: 666-77. http://dx.doi.org/10.1128/MCB.01716-07

[15] KATANASAKA Y, ISHII T, ASAI T, NAITOU H, MAEDA $\mathrm{N}$, et al. Cancer antineovascular therapy with liposome drug delivery systems targeted to BiP/GRP78. Int J Cancer 2010; 127: 2685-98. http://dx.doi.org/10.1002/ijc.25276

[16] FRIBLEY AM, MILLER JR, BROWNELL AL, GARSHOTT $\mathrm{DM}, \mathrm{ZENG} \mathrm{Q}$, et al. Celastrol induces unfolded protein response-dependent cell death in head and neck cancer. Exp Cell Res 2015; 330: 412-22. http://dx.doi.org/10.1016/j. yexcr.2014.08.014

[17] LIN ML, CHEN SS, LU YC, LIANG RY, HO YT, et al. Rhein induces apoptosis through induction of endoplasmic reticulum stress and $\mathrm{Ca} 2+-$ dependent mitochondrial death pathway in human nasopharyngeal carcinoma cells. Anticancer Res 2007; 27: 3313-22. 